\title{
POR UMA SOCIOLOGIA DO CUIDADO: REFLEXÕES PARA ALÉM DO CAMPO DA SAÚDE
}

\author{
FOR A SOCIOLOGY OF CARE: \\ REFLECTIONS BEYOND THE FIELD OF HEALTH
}

\begin{abstract}
Octávio Augusto Contatore (iD) (https://orcid.org/0000-0003-3486-7924) ${ }^{1}$, Ana Paula Serrata Malfitano (http://orcid.org/0000-0002-0502-3194) ${ }^{2}$,Nelson Filice de Barros ${ }_{\text {(https://orcid.org/0000-0002-5404-1516) }}^{1}$
\end{abstract}

${ }^{1}$ Universidade Estadual de Campinas, Faculdade de Ciências Médicas, Departamento de Saúde Coletiva, Campinas, São Paulo, Brasil.

<oacontatore@yahoo.com.br>

\author{
${ }^{2}$ Universidade Federal de São Carlos, Departamento de Terapia Ocupacional, Programa de Pós-Graduação \\ em Terapia Ocupacional, São Carlos, São Paulo, Brasil.
}

Resumo Embora a pesquisa sobre o cuidado esteja predominantemente associada ao campo da saúde, os estudos sociológicos ressaltam que os elementos que o influenciam não se restringem a ele, na medida em que é a expressão de apoio social intenso e atributo fundamental para a manutenção da vida coletiva. Objetivou-se hipervisibilizar a dimensão sociológica do cuidado para problematizar sua transposição como fator necessário à vida social para uma ação técnica de saúde. Realizouse uma revisão sistemática de literatura, entre 2003 e 2013, na Biblioteca Virtual em Saúde, PubMed, Scopus, Embase, Web of Science, Francis (Ovid), ProQuest Central, Academic Search Premier (EBSCOhost), Jstor e Sage, com o unitermo cuidado e variantes. De 262 artigos identificados, foram selecionados 15 sobre as dimensões sociais do cuidado. Observou-se que o cuidado aludiu à solidariedade e qualidade da interação entre sujeitos nas microrrelações e nas macroestruturas, diferenciando-se de sua aplicação técnica/tecnológica em saúde; e também que é mais abrangente defini-lo sociologicamente do que pela especificidade de um ato, pois inclui dimensões de solidariedade, respeito, zelo e ajuda mútua para a conservação da vida. Concluiu-se que uma abordagem sociológica do cuidado é necessária, contribuindo inclusive para o campo da saúde. Palavras-chave sociologia; cuidados; cuidados de saúde; apoio social.
Abstract Although research on care is predominantly associated with the health field, sociological studies emphasize that the elements that influence the health field are not restricted to it, insofar as it is the expression of intense social support and a fundamental attribute for the maintenance of collective life. The objective of the present study was to hypervisualize the sociological dimension of the care to problematize its transposition as a necessary factor to the social life for a technical action of health. A systematic literature review was conducted between 2003 and 2013 in the Virtual Health Library, PubMed, Scopus, Embase, Web of Science, Francis (Ovid), ProQuest Central, Academic Search Premier (EBSCOhost), and Jstor and Sage databases, with the single term care and its variants. Of the 262 articles identified, 15 on the social dimensions of care were selected. It was observed that care referred to the solidarity and quality of the interaction between subjects in micro-relationships and macro-structures, differing from its technical/technological application in health. It was also observed that it is more comprehensive to define care sociologically than by the specificity of an act, for it includes dimensions of solidarity, respect, zeal, and mutual help for the preservation of life. It was concluded that a sociological approach to care is necessary, even contributing to the health field.

Keywords sociology; care; health care; social support. 


\section{Introdução}

Embora o cuidado seja predominantemente associado ao campo da saúde, os estudos sociológicos ressaltam que os elementos que o influenciam não se restringem a esse campo, na medida em que as ações de cuidado são compreendidas pela perspectiva de um apoio social fundamental para a manutenção da vida coletiva (Fine, 2005; Pescosolido, 2006; Carpentier, 2013), o que ultrapassa os limites do campo da saúde.

Na saúde, a noção de cuidado vem sendo estudada em diferentes perspectivas, podendo ser exemplificadas: em pragmática, da clínica ampliada, gerencial, filosófica, emancipadora, política, sociológica e cultural (Contatore, Malfitano e Barros, 2017). Contudo, o enfoque dos estudos do campo da saúde relaciona-se, em sua maioria, a diagnósticos, tratamento, prevenção e cura de doenças, com base em um conhecimento biológico (Camargo Jr., 2005; Campos e Bedrikow, 2014). Apesar de uma já consolidada discussão acerca da insuficiência do modelo biomédico (Camargo Jr., 2005; Tesser, 2007) e da recolocação da noção de 'taciticidade' no conceito de cuidado (Barros, 2015), a racionalidade médica científica (Luz, 2000) predomina em tais estudos.

Nos estudos sociológicos, o tema cuidado não ganhou um lugar prioritário como conceito de relevância para a compreensão da constituição social, contudo é importante destacar pesquisas que buscam a visibilidade de tal discussão, notadamente por meio da associação com outras temáticas, como trabalho e gênero (Georges e Santos, 2014). Com pouco destaque no mundo acadêmico até o final do século XX, foi principalmente compreendido como o resultado de um comportamento de gênero pertencente às mulheres, notadamente na esfera familiar. Novos estudos demonstrando a emergência desse tema foram impulsionados em decorrência de significativas mudanças sociais, tais como o aumento da participação das mulheres no mercado de trabalho, a passagem das ações de cuidado da esfera privada familiar para o espaço público, a crise de oferta de atenção pelos sistemas de saúde e o questionamento da predominância do modelo biomédico como referência para a execução das ações técnicas de cuidado (Fine, 2005). Essas mudanças foram impulsionando uma nova abordagem do tema pela filosofia, pela sociologia e pela antropologia, trazendo para a arena o debate acerca do cuidado como elemento influenciador das relações sociais.

Tal debate despolarizou a esfera de cuidado às mulheres e suas casas, sendo que os novos estudos sociológicos referenciaram o cuidado como uma expressão de apoio social intenso, um atributo fundamental para a manutenção da vida e, conjuntamente com a solidariedade social, em vista do reconhecimento da vulnerabilidade do corpo, a base para o desenvolvimento de outros componentes essenciais da vida social (Fine, 2005). 
Porém, os estudos sobre o cuidado no campo da saúde, voltados em sua ênfase à formalização das ações técnicas de assistência efetuadas pelos profissionais da área, predominaram quando comparados aos estudos do cuidado no campo da sociologia (Ayres, 2004; Erdmann et al., 2004; Silva et al., 2014; Lopes, 2015; Almeida, Marin e Casotti, 2017). Ou seja, embora o cuidado seja um elemento intrínseco à vida humana, o imaginário social, bem como os debates acadêmicos brasileiros acerca da temática, relaciona-o predominantemente ao campo da saúde, em vez de compreender o cuidado por meio de sua dimensão sociológica e pertencente, entre outros fatores, às questões de saúde.

Nesse debate, o objetivo do estudo que originou este artigo, o qual foi desenvolvido em dissertação de mestrado realizada em 2016 na Faculdade de Ciências Médicas, no Departamento de Saúde Coletiva, da Universidade Estadual de Campinas (Contatore, 2016), foi hipervisibilizar a dimensão sociológica do cuidado para problematizar sua transposição de fator necessário à vida social para uma ação técnica de saúde. Para essa discussão, apoiou-se na busca de artigos sobre o cuidado, com enfoque na produção sociológica, indexados na literatura acadêmica. Realizou-se uma revisão sistemática da literatura, com foco nos trabalhos de cunho sociológico. Os resultados foram detalhadamente apresentados para suportar uma discussão sobre as relações do cuidado com o capitalismo financeiro, as disparidades sociais e a sua redução à dimensão técnica e tecnológica, elementos presumíveis de discussão em uma sociologia do cuidado.

\section{Procedimentos metodológicos}

Foi realizada uma revisão sistemática da literatura com os descritores "Assistência à saúde" or "Prestação de Assistência à Saúde" or "Prestação de Cuidados de Saúde" or "Prestação de Assistência Odontológica" or "Prestação de Cuidados Odontológicos" or "Cuidados de Saúde" or "Cuidados de Assistência à Saúde" or "Rede de Cuidados Continuados de Saúde" and "Sociologia Médica" na Biblioteca Virtual em Saúde e seus correspondentes em inglês "Delivery of Health Care" or "Health Care Delivery" or "Health Care Systems" or "Delivery of Dental Care" or "Health Care" and "Sociology Medical" nas bases de dados que contemplam artigos da área da saúde: PubMed, Scopus, Embase, Web of Science, Francis (Ovid), ProQuest Central e Academic Search Premier (EBSCOhost). E com os descritores Care or Health or Healthcare and (abstract) Sociology nas bases de dados que contemplam artigos das ciências sociais: Jstor e Sage.

A revisão sistemática da literatura é um recurso metodológico que tem sido utilizado com a finalidade de compilar resultados acerca dos estudos já realizados sobre uma determinada temática, buscando sintetizar o estado da 
arte acerca de um conhecimento. Para tanto, visa à produção de "sínteses concisas sobre uma questão específica, já que é conduzida de modo a identificar, analisar e reunir dados procedentes de fontes diversas e independentes sobre o mesmo assunto" (Gonçalo et al., 2012, p. 105).

É preciso ressaltar que uma revisão sistemática da literatura atinge aquilo que os descritores e as bases de dados permitem alcançar, o que não necessariamente contempla todo o campo a ser pesquisado. No presente estudo, voltou-se apenas aos artigos disponíveis aos pesquisadores brasileiros pelo Portal de Periódicos da Coordenação de Aperfeiçoamento de Pessoal de Nível Superior (Capes), resultando no não acesso à integralidade do universo de artigos sobre a temática. Dessa maneira, a análise realizada dos achados é uma leitura parcial do objeto de estudo.

A pesquisa foi efetuada em outubro de 2014, e os critérios de inclusão foram: artigo ter sido publicado no período entre 2003 e 2013; ser um artigo científico indexado; ter sido publicado em português, espanhol ou inglês; ter um dos descritores no título ou no resumo. Já os critérios de exclusão foram: artigos que não fossem de acesso aberto ou não disponibilizados no Portal de Periódicos da Capes, artigos de revisão de literatura, carta, editorial, dissertação ou tese, artigos que estivessem em periódicos que não oferecessem acesso integral aos textos. A Tabela 1 relaciona o número de artigos encontrados em cada base de dados.

Tabela 1

\begin{tabular}{ll}
\hline Número de artigos encontrados em cada base de dados & \\
\hline Base de dados & Total de artigos selecionados em cada base de dados \\
\hline Biblioteca Virtual em Saúde & 20 \\
PubMed & 112 \\
Scopus & 39 \\
Embase & 221 \\
Web of Science & 2 \\
Francis (Ovid) & 171 \\
ProQuest Central & 121 \\
Academic Search Premier (EBSCOhost) & 35 \\
Jstor & 99 \\
Sage & 852 \\
Total &
\end{tabular}

Na primeira fase foi feita a busca de artigos em cada base de dados, filtrando-se apenas com a utilização de aspas pelos descritores, contabilizando 852 textos. Na segunda etapa, removeram-se os textos duplicados e aplicaramse os critérios de inclusão elencados, restando 262 textos. Na terceira etapa, fez-se uma leitura na íntegra dos artigos, eliminando sessenta que não tinham relação com a temática do cuidado. Na quarta etapa, classificaram-se os 202 artigos em oito áreas temáticas: estudos sociológicos sobre saúde, doença e 
aplicação do cuidado; análise, mudanças ou reformulação dos Sistemas Nacionais de Serviços de Saúde (SNSSs); profissionais e suas entidades refletindo sobre suas práticas de atenção à saúde; determinantes sociais: disparidades e desigualdade no acesso aos sistemas de saúde; atenção à saúde pelo modelo biomédico; uso de tecnologias voltadas para o aperfeiçoamento da atenção à saúde; fatores econômicos influenciando a atenção à saúde; uso de práticas não biomédicas na atenção à saúde. Nesta classificação, identificaram-se 23 artigos na área 1, "Estudos sociológicos sobre saúde, doença e aplicação do cuidado". Na quinta etapa, procedeu-se à nova leitura dos artigos da área 1 buscando aqueles que enfocassem centralmente discussões do campo da sociologia e da saúde sobre o cuidado, o que resultou na seleção de 15 textos para análise. O esquema para visualização do processo de seleção dos artigos está sintetizado na Figura 1.

\section{Figura 1}

Processo de seleção dos artigos

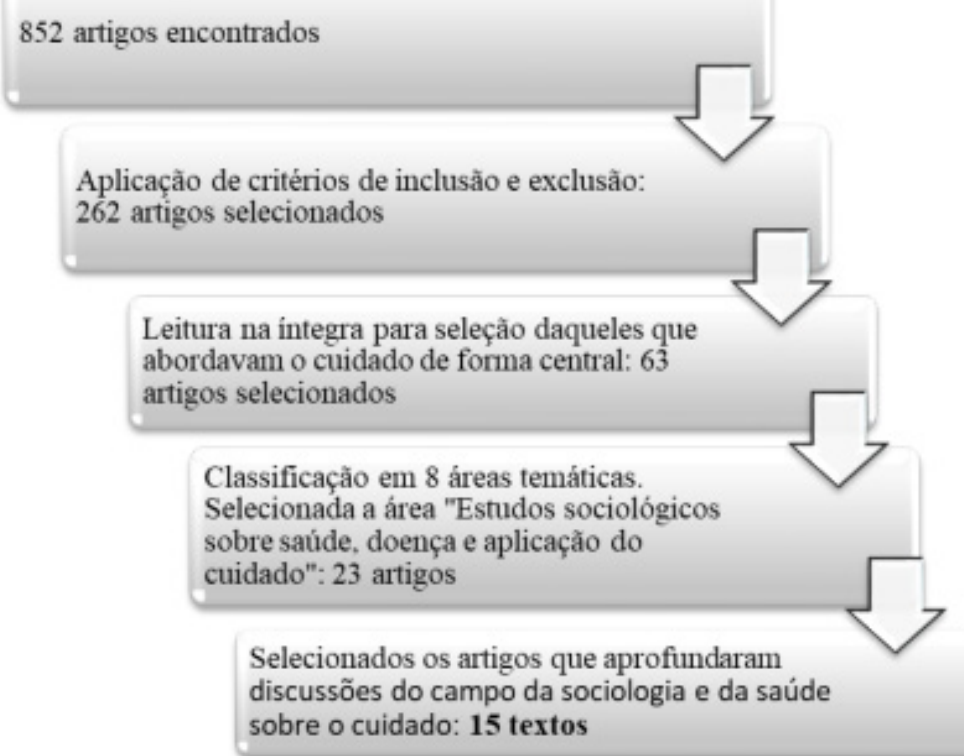

Fonte: Os autores.

O grupo de 15 artigos $^{4}$ foi composto pelas seguintes referências: Carpentier (2013); Carpentier e Grenier (2012); Clements et al. (2008); Denier (2008); Dovidio et al. (2008); Ferlander (2007); Fine (2005); Hanlon e Halseth (2005); Kim, Farmer e Porter (2013); Noone e Stephens (2008); Oh (2013); Pescosolido (2006); Pavloková e Prusvic (2010); Samitt (2004); Spates (2012). 
Com base no levantamento efetivado, foi realizada uma análise de conteúdo temática da literatura encontrada, buscando o levantamento de categorias que possibilitassem uma perspectiva compreensiva dos estudos realizados nesse campo. A análise temática dedica-se ao levantamento de categorias, de escolha dos autores, visando à contribuição acerca das discussões de um determinado fenômeno (Minayo, 2007).

\section{Resultados}

Nos artigos selecionados, o cuidado foi referido por diferentes abordagens e observou-se maior predominância da discussão das seguintes temáticas: avaliação do conceito de cuidado e seu lugar nos estudos sociológicos (Fine, 2005); cuidado na interface com o campo da saúde focalizando a importância do apoio social/redes sociais para o resultado das ações de cuidado (Pescosolido, 2006; Carpentier e Grenier, 2012; Carpentier, 2013); interferência das disparidades sociais no acesso ao cuidado (Ferlander, 2007; Dovidio et al., 2008; Noone e Stephens, 2008; Spates, 2012); e fatores econômicos influenciando a atenção à saúde, especificamente os sistemas público e privado na sua inter-relação com o cuidado (Fine, 2005; Hanlon e Halseth, 2005; Clements et al., 2008; Denier, 2008; Pavloková e Prusvic, 2010; Kim, Farmer e Porter, 2013; Oh, 2013; Samitt, 2004).

As três temáticas destacadas dos textos demonstraram não haver uma descrição linear sobre qual o lugar do cuidado em uma perspectiva da sociologia - tendo como fonte as bases de dados relacionadas à sociologia, uma vez que o seu conceito atravessa temas e níveis de análise, pois liga microinterações e macroestruturas e reúne aspectos formais e informais relacionados à vida social, em vez de tratá-los como temas especializados e distintos de apenas um campo (Fine, 2005).

\section{Sociologia do cuidado: visibilidades e invisibilidades}

Na primeira temática, o cuidado é abordado conceitualmente e sua localização é definida nos estudos sociológicos. Também se contextualiza sua importância para a manutenção da estrutura social, mediante a solidariedade entre sujeitos em busca de preservar a vida em diferentes momentos, da infância à velhice (Fine, 2005).

Até o final do século XX, ignorou-se a importância fundamental do cuidado para a vida social, pois ele foi entendido como responsabilidade familiar, presente em relações de parentesco e casamento em que a devoção e o dever pessoal eram comportamentos singulares pertencentes às mulheres (Fine, 2005). Entretanto, mudanças sociais, econômicas e culturais têm promovido alterações na vida pessoal e doméstica (Castel, 1994), por meio da mudança 
do lugar da mulher na sociedade (Biasoli-Alves, 2000). A individualização da vida social ligou objetivos pessoais a mudanças sociais, sendo que o cuidado, longamente construído como um dever e responsabilidade do feminino, passou por consideráveis transformações. Assim, a mudança social do lugar da mulher, de alguém que vive uma vida para os outros, para o direito à escolha de buscar uma vida própria reformulou o lugar da categoria cuidado nos estudos em sociologia (Fine, 2005).

Portanto, o cuidado passou por um percurso histórico de invisibilidade para visibilidade na sociologia. A principal investigação encontrada nesse tópico, a qual realiza uma síntese conceitual sobre a emergência dos estudos em sociologia sobre o cuidado, afirma que nenhum dos principais teóricos contemporâneos identificou uma sociologia do cuidado (Fine, 2005), embora seja necessário pontuar a existência de relevantes estudos no campo da sociologia sobre o cuidado, ou care, com destaque para a discussão acerca do lugar da mulher (Georges e Santos, 2014). Entretanto, a temática deveria ser mais reconhecida e estudada pela centralidade do cuidado na teoria social e política, de modo a contribuir para uma análise social mais avançada.

\section{O que influencia o cuidado em saúde}

A segunda temática está relacionada aos textos (Pescosolido, 2006; Carpentier e Grenier, 2012; Carpentier, 2013) que discutiram especificamente o cuidado em saúde, aqui considerado como uma das possíveis dimensões do cuidado. A relação entre os cuidadores formais e informais destacou a influência dos contextos sociais nas ações de cuidado empreendidas na atenção à saúde. Cuidador formal e informal foram utilizados aqui seguindo a linguagem empregada nos textos, os quais referem como cuidador formal aquele que teve uma qualificação específica no campo da saúde; e informal a pessoa sem qualificação técnica que exerce a atividade de cuidado sob um contrato de trabalho ou não. O cuidado foi abordado, principalmente, do ponto de vista dos atores sociais que são cuidadores ou receptores de cuidado e de sua interação com os profissionais de saúde (Carpentier e Grenier, 2012). Tal discussão é também abordada na sociologia do trabalho, notadamente em estudos em outros países. Nessa dimensão, os estudos sociológicos e biomédicos utilizaram as redes de apoio sociais para compreender os processos de saúde-doença e demonstraram, no nível organizacional, que o principal determinante do tipo de cuidado (familiar, por exemplo) ou resultado do cuidado não é a modalidade de tratamento, mas sim a natureza das redes sociais baseadas na organização entre provedores (Pescosolido, 2006; Carpentier e Grenier, 2012).

Os problemas de saúde foram realocados para uma dinâmica de relações sociais e redes de atenção incluindo a dimensão social da esfera médica e as 
estratégias de ação dos diversos atores envolvidos em processos de tomada de decisão (Carpentier, 2013; Carpentier e Grenier, 2012), já que a relação não consiste simplesmente entre um único indivíduo e um profissional de saúde. Ela se dá entre uma pessoa que está localizada dentro de uma miríade de relações - às vezes de suporte, outras vezes afetadas por rivalidades e conflitos familiares - e um profissional de saúde que também faz parte de uma complexa rede de relações, incluindo lutas intra e interprofissionais (Carpentier, 2013).

Os textos desse segmento pontuaram a importância de qualificar um cuidado para que, na sua efetivação, não só abranja a esfera profissional mas também valorize a participação de todos os atores sociais envolvidos. Dessa maneira, o seu foco não esteve na especialização médica ou na tecnologia aplicada, especialmente por problematizar que sistemas de cuidados teriam de ser concebidos dentro de uma organização social ativa e em evolução, compostos dos numerosos atores envolvidos na sua prestação (Pescosolido, 2006; Carpentier, 2013).

O cuidado, visto como resultado das redes sociais dos sujeitos, possibilitou a afirmação de que o atual sucesso da ciência biomédica está relacionado a uma mudança de olhar, do microscópio para o ambiente. Dessa maneira, apontou-se o quão surpreendente foram, para o modelo biomédico, os achados que sugerem melhor recuperação da saúde via uma assistência médica menos sofisticada e com menos suporte tecnológico, porém com maior nível de interação entre os envolvidos (Pescosolido, 2006). Em outras palavras, como se efetivam as contribuições de uma análise sociológica ao campo da saúde.

Contrariamente ao modelo da medicina baseada em evidências (MBE) e sua determinação em protocolar as ações de cuidado com base na evidência clínica, foi compreendida a distinção entre a eficácia (efficacy) dos tratamentos, que funcionam estritamente dentro de ensaios clínicos controlados, e a eficiência (effectiveness), ou seja, aquilo que funciona no 'mundo real' (Pescosolido, 2006) - o que marca uma mudança nas explicações clínicas em relação a contextos de cuidado. Perderam-se as expectativas de que os tratamentos têm efeito idêntico e de que os indivíduos reagem da mesma forma, e deu-se lugar às investigações sobre os impactos social e psicológico na perspectiva de cada indivíduo, o efeito da cultura e do clima no local do tratamento, a importância do apoio da comunidade e a valorização das estratégias leigas (Pescosolido, 2006; Carpentier e Grenier, 2012). O que vem demonstrar a fragilidade implícita da ideia de que o cuidado e qualquer resultado positivo advindo de suas ações podem ser representados apenas pela esfera técnica/ tecnológica da assistência à saúde, na medida em que se constatou a influência das redes sociais dos sujeitos, ou seja, da qualidade do apoio entre as pessoas envolvidas, fazendo um movimento contrário à hipervalorização do cuidado técnico/tecnológico descontextualizado das relações ali envolvidas. Ou seja, explicitou-se a relação entre cuidado e sociedade. 


\section{Disparidades sociais, questões econômicas e o cuidado em saúde}

A terceira temática discutiu a influência das disparidades sociais para o acesso ao cuidado em saúde (Ferlander, 2007; Dovidio et al., 2008; Noone e Stephens, 2008; Spates, 2012) e problematizou quais eram as limitações de acesso para determinado grupo social em razão de fatores culturais, da desigualdade social e do preconceito vivido. A obtenção de cuidado foi associada aos temas: capital social, classe social, conceito de masculinidade e racismo. Abordou também a relação entre o cuidado e fatores econômicos (Fine, 2005; Hanlon e Halseth, 2005; Clements et al., 2008; Denier, 2008; Pavloková e Prusvic, 2010; Kim, Farmer e Porter, 2013; Oh, 2013; Samitt, 2004), enfocando a influência das questões financeiras nos sistemas público e privado para o acesso à saúde pela população. Estes estudos expuseram alguns fatores considerados causais do aumento dos custos em saúde, tais como o envelhecimento da população, o desenvolvimento tecnológico, a expansão demográfica e a falta de recursos financeiros para custear o cuidado. No entanto, compreenderam que a diminuição dos recursos econômicos na área da saúde é desfavorável ao cuidado e criticaram medidas utilizadas que justificam a diminuição do financiamento do setor saúde.

Dois dos trabalhos (Ferlander, 2007; Noone e Stephens, 2008) focaram nas dificuldades de pessoas de classe social com menor recurso econômico e acesso aos bens sociais para a obtenção de cuidado na atenção à saúde, compreendendo a dimensão sociocultural envolvida. Dessa maneira, formulou-se a hipótese de que uma distribuição equilibrada de capital social, abordado como rede e suporte sociais, bem como a informação e cultura acessadas, provavelmente beneficiaria os indivíduos a ter melhor acesso aos recursos de cuidado, como aqueles oferecidos pelos serviços de saúde pública (Ferlander, 2007). Foi enfocado especificamente que o ideal de masculinidade, especialmente nas classes sociais com menor poder aquisitivo, repercute no entendimento de suas necessidades de saúde, na compreensão e incompreensão da linguagem médica e na busca por cuidados (Noone e Stephens, 2008). Com isso, o capital social seria um recurso individual e comunitário acessado por meio de redes sociais que possibilitaria alcançar níveis de apoio, troca de informações, tolerância e acessibilidade (Ferlander, 2007). Portanto, nessa perspectiva, melhorar as condições de cuidado em saúde implica ampliar a dimensão sociocultural de diferentes grupos.

Outros dois trabalhos discutiram a influência da discriminação racial (Dovidio et al., 2008; Spates, 2012) prejudicando a qualidade dos resultados do cuidado em saúde. Os textos pontuaram que, por exemplo, nos Estados Unidos da América, a exclusão de pesquisas a respeito das mulheres negras na saúde mental, do preconceito, do desconhecimento cultural e de condições opressivas a essa população contribuiu para a ocorrência de erros que 
repercutiram em maior incidência de pessoas diagnosticadas com problemas mentais nessa população, como também nas atitudes discriminatórias de alguns profissionais em relação à população negra - o que desfavoreceu uma relação de confiança entre as duas partes.

Na perspectiva econômica, os textos (Fine, 2005; Hanlon e Halseth, 2005; Clements et al., 2008; Denier, 2008; Pavloková e Prusvic, 2010; Kim, Farmer e Porter, 2013; Oh, 2013; Samitt, 2004) abordaram a crise nos sistemas de saúde e compreenderam o cuidado pelo viés das questões financeiras necessárias para efetivá-lo na assistência à saúde. Entenderam, portanto, que sua ação compreende a dimensão econômica, política e gerencial de se cuidar coletivamente da saúde da população. O que remete à dimensão societária do cuidado, descrita por Cecílio (2011), que trata do papel do Estado na produção de políticas públicas em geral e nas de saúde em particular, ou à abordagem de Tronto (2007), que relaciona cuidado e democracia.

Embora salientem que o aumento demográfico e o envelhecimento da população elevam os custos para aplicação do cuidado (Pavloková e Prusvic, 2010), os autores apontam a ambivalência no discurso da redução dos custos, na medida em que eles não são determinados apenas pelas demandas concretas de insumos e serviços pela população (Denier, 2008), mas também pelos interesses políticos e econômicos que atuam na determinação do que é prioridade e daí o que é 'alto' custo para a manutenção do cuidado à saúde (Oh, 2013).

Nessa perspectiva da prioridade de investimentos em saúde, foi pontuado que o envelhecimento da população na zona rural exige um aumento de recursos financeiros para custear o cuidado, uma vez que não se mantém o padrão de assistência à saúde efetuado nos centros urbanos (Hanlon e Halseth, 2005). Enfatizou-se a impossibilidade futura de manter o financiamento dos sistemas de saúde da Europa, em razão do envelhecimento da população e da expansão demográfica (Pavloková e Prusvic, 2010). Também se discutiu que os benefícios econômicos advindos das práticas implementadas de reduções de custo dos sistemas de saúde levaram a uma falsa economia, uma vez que a superlotação e a falta de pessoal tiveram um efeito negativo sobre a segurança do paciente e a qualidade do atendimento, o que resultou em novos problemas de saúde, que geraram aumento nos custos do cuidado (Clements et al., 2008).

Com uma posição ideológica contra-hegemônica à proposta neoliberal de desmonte dos sistemas nacionais de saúde (Pires e Demo, 2006), afirmou-se que a melhora da prestação de cuidados em saúde no mundo, para as populações mais pobres, não era um dreno para a economia, mas sim resultaria em melhora econômica (Kim, Farmer e Porter, 2013) - uma vez que, se bem projetados, os sistemas de atenção à saúde baseados na prestação de serviços de alto valor seriam recursos poderosos para o desenvolvimento econômico. Dessa forma, os autores analisaram o cuidado em saúde em uma perspectiva 
macrossocial, assinalando as desigualdades sociais e enfocando os necessários investimentos financeiros para sua efetivação.

\section{Discussão}

Os trabalhos em análise contemplaram o principal objetivo da pesquisa de hipervisibilizar e compreender a dimensão sociológica dada ao substantivo cuidado, buscando seu destaque na discussão brasileira. Pode-se afirmar que, nesses estudos, o cuidado refere-se à interação solidária entre as pessoas e a construção dos seus sentidos, o que não está diretamente ligado ao uso aplicado que lhe é característico no campo da saúde, mesmo quando a discussão sobre o tema perpassa tais práticas. O cuidado foi descrito como uma expressão de apoio social intenso (Fine, 2005), algo

como um tipo de prestação de ajuda que repousa (...) nos intercâmbios, obrigações e padrões de reciprocidade entre indivíduos, grupos, famílias e instituições, portando significados para os atores neles envoltos, nas suas respectivas experiências cotidianas e contextos (Canesqui e Barsaglini, 2012, p. 1.104).

Trata-se da relevância de temáticas em torno do apoio social, das redes sociais de suporte, da reciprocidade e do gênero como componentes essenciais dessa discussão.

A primeira temática está relacionada a uma descrição geral do lugar atribuído pela sociologia ao cuidado. Historicamente, sua relativa marginalidade nos estudos sociológicos foi justificada pelo lugar de pequena qualificação social das ações de cuidar na sociedade. A naturalização do cuidado, associando-o diretamente ao gênero feminino, colocou-o na mesma posição social inferiorizada tradicionalmente atribuída às mulheres e consequentemente ao seu trabalho. Essa inter-relação entre cuidado, gênero feminino e posição social vai ao encontro do estudo clássico de Simone de Beauvoir (1980), resultante de seu livro intitulado O segundo sexo, publicado originalmente em 1949. A autora disserta sobre como a condição feminina é uma construção social baseada numa visão masculina, de que a mulher é um 'outro' de menor status social e seu trabalho não é visto como essencial, envolvendo pouco conhecimento intelectual e valor econômico. Importante ressaltar que, para Beauvoir, os atributos considerados femininos não são natos, mas aprendidos culturalmente, pois, nas suas palavras, "Ninguém nasce mulher: torna-se mulher" (Beauvoir, 1980, v. II, p. 9).

Como consequência dos processos de mudança de lugar da mulher na sociedade (Biasoli-Alves, 2000), o cuidado foi deslocado para o domínio público, e a disponibilidade de prestação de cuidados transformou-se num aspecto cada 
vez mais controverso da vida moderna (Fine, 2005), o que estabeleceu uma nova geografia dos cuidados informais, embora com a ainda centralidade do lugar da mulher em diferentes ações de cuidado, desde as tarefas domésticas, por meio do trabalho de empregadas (Georges e Santos, 2014), aos cuidados das crianças e dos idosos (Debert, 2014). Tais fatores, em meio à crise dos sistemas de assistência social e de saúde nas sociedades mais avançadas, organizaram uma nova relação entre prestadores de serviços de cuidado, em saúde ou em outras áreas, e seus beneficiários.

O trabalho de cuidar, antes majoritariamente ignorado como campo de estudo e relegado ao anonimato acadêmico, em razão de comportar elementos culturalmente considerados pertencentes ao mundo feminino, ao adentrar a esfera pública passou a ser alvo de preocupação da sociologia, de forma que a própria sociologia também foi deslocada para áreas menos valorizadas pela tônica racional da pesquisa científica, característica do mundo cultural masculino e predominante na ciência em gênero, número e grau (Costa e Sardenberg, 2002). A sociologia do trabalho passa a adentrar nos estudos a dimensão dos cuidadores e seu lugar de menor qualificação social para exercer o trabalho de cuidar (Lallement, 2014) - o que remete à crítica feita por Santos (2002) à racionalidade do pensamento científico contemporâneo e à sua limitação em considerar importante e explicar a amplitude e a variedade das experiências sociais presentes no mundo. Tal pensamento, no entanto, é tensionado a mudar pela influência do poder social como indicador privilegiado dos dilemas e das contradições que alimentam as transições paradigmáticas.

Os estudos sociológicos sobre o care (Guimarães, Hirata e Sugita, 2011; Sorj, 2014) possivelmente podem representar o lugar emergente que o cuidado passou a ter na sociologia (Fine, 2005). Entretanto, ao partir desse histórico sobre o cuidado ao outro como atributo familiar feminino, o campo da sociologia do trabalho faz um recorte nos cuidadores desassociados da profissionalização, o que acaba por excluir, por exemplo, os profissionais de nível superior na saúde. Nenhum artigo sobre o que vem sendo denominado de care apareceu nas buscas dessa revisão, em razão de os artigos abordarem o cuidado mais na esfera das relações de trabalho, ou seja, o cuidado como uma forma de trabalho formal ou informal e tratado pela sociologia do trabalho. Os estudos dessa área de pesquisa problematizam o baixo valor existente na inter-relação entre trabalho, gênero e cuidado, tanto na esfera domiciliar como na profissional. Seu objeto são as relações de trabalho de cuidadoras ou cuidadores, sejam remunerados ou não, qualificados ou não, que assistem idosos, crianças ou pessoas com necessidades especiais. Também buscam entender o universo dos profissionais da área da saúde, mais especificamente auxiliares de enfermagem ou enfermeiros, que cumprem as funções de cuidado que exigem maior atenção afetiva e intimidade. O significado de care, traduzido para o português como cuidar do outro, conota um amplo campo de ações e 
atitudes nas relações de cuidado e pode expressar a ideia de solicitude, inquietude afetiva pelo outro (Guimarães, Hirata e Sugita, 2011; Sorj, 2014).

Na perspectiva dos estudos sobre o care, quando o cuidado sai da esfera doméstica e passa a ser profissionalizado não muda a sua desvalorização social, independentemente do gênero de quem o aplica, lembrando que permanece a predominância de mulheres. O seu valor econômico e social está diretamente relacionado às características do trabalho exercido. As ações de cuidado, quando estão ligadas às emoções, à intimidade e ao contato com os fluidos excretados pelo corpo, são menos remuneradas e valorizadas socialmente e exercidas por mulheres (em sua maioria), por pessoas pobres - o que é extremamente comum no Brasil, mas também presente em países europeus pela participação de imigrantes (Molinier, 2014) e grupos étnicos desfavorecidos economicamente (anteriormente escravos). Já aquelas ações que envolvem menos intimidade são mais pontuais (não exigem uma longa dedicação) e têm maior especialização técnica; são vistas com maior status social e alcançam melhor remuneração (Fine, 2005; Guimarães, Hirata e Sugita, 2011; Epele, 2012; Sorj, 2014). Todavia, a abordagem central se dá na necessária discussão sobre o trabalho e as precárias relações de exploração ali estabelecidas.

Em síntese, o cuidado caminha para maior visibilidade nos estudos sociológicos, mas ainda com um recorte temático importante no âmbito do trabalho. Os estudos sobre dádiva (Caillé, 2014), reciprocidade (Murray et al., 2017) e empatia (Hollan, 2008) também têm sido desenvolvidos, enriquecendo o debate acerca do cuidado.

O conceito de cuidado nesta pesquisa foi abordado na sua expressão social mais preliminar, implícito em qualquer ação voltada para a conservação da vida, realizado por qualquer membro da sociedade. Se considerarmos o cuidado como um elemento básico de toda a vida social, ou uma expressão de apoio social intenso, da infância à velhice, é imprescindível sua compreensão como resposta social necessária à vulnerabilidade, incapacidade e incompletude de corpos individuais em diferentes momentos no curso da vida (Fine, 2005).

Especificamente no campo da saúde, outros estudos afirmaram que as redes sociais são fundamentais no cuidado em saúde (Pescosolido, 2006; Carpentier e Grenier, 2012; Carpentier, 2013), por serem compreendidas como a articulação social em torno da operacionalização de diferentes serviços e atores sociais, para as quais a densidade da constituição da rede depende da relação, da quantidade e da qualidade de cada ator com os demais componentes (Gomide e Grossetti, 2010).

A constatação da força do cuidado no interior das redes sociais, envolvendo vários atores, fortalece o valor da sua 'dimensão social originária', anterior ao seu posisionamento especializado no campo da saúde. Ou seja, enfoca a necessidade da compreensão do cuidado como elemento pertencente à esfera social e não como sinônimo de ação em saúde. Tal dimensão está 
na perspectiva apresentada por Barros (2015), na qual o cuidado é resultante do processo de socialização e pode ser compreendido como um fazer tácito do ser humano, sendo que ao mesmo tempo que é uma prática individual, é construída coletivamente. O que remete à ideia, presente nos trabalhos de Canguilhem (2002), Foucault (1977) e Illich (1975), de que clínico era aquele que se colocava ao pé da cama, ao lado da pessoa doente noite e dia. Esses autores localizaram, a partir da criação da clínica médica, o momento em que o cuidado começou a ser concebido como aplicação técnica por um profissional especializado e compreenderam o impacto causado pelo saber científico na percepção social do que é o cuidado.

No campo das práticas de cuidado em saúde, as pesquisas empreendidas, ao analisarem os processos envolvidos nos adoecimentos, buscaram compreender quais são os limites das abordagens biomédicas e construíram abordagens integradas que ajudaram entender quem fica doente, quem busca tratamento, quem se recupera e por que isso acontece. As conclusões de que os resultados sobre saúde, doença e cura são moldados por fatores extramédicos ou extratratamentos (Pescosolido, 2006) não eliminam a relevância de técnicas e tecnologias de qualidade para o atendimento, em nenhuma hipótese, porém assinalam que elas, isoladamente, não respondem aos elementos que atuam no cuidado em saúde. Portanto, os estudos de cunho sociológico trazem novos elementos para repensar e reorientar os cuidados em saúde.

Podemos identificar um movimento nas diretrizes brasileiras de organização da assistência em saúde que vai ao encontro da proposição de redes, como as redes de atenção em saúde - RAS (Brasil, 2010) - e as redes de atenção psicossocial - RAPS (Brasil, 2011). O que os estudos aqui analisados adicionam é que os elementos em diálogo estão além dos serviços formais que compõem as redes, já que a assistência deve incorporar as redes informais, ou seja, os elementos de sua vida social, de cada sujeito, como componentes do cuidado em saúde.

Em oposição ao discurso atual de falta de recursos financeiros para a manutenção do cuidado, desenvolveram-se reflexões que contrariam a hipótese de que a dificuldade em realizar um equilíbrio aceitável entre eficiência, justiça e cuidado é originada da escassez de recursos (Denier, 2008). Sobretudo porque essa argumentação crítica baseia-se na reflexão sobre o avanço exponencial da tecnologia médica e a crescente medicalização da vida, que trouxe mudanças importantes no modo de cuidar, em detrimento de um cuidado autêntico, respeitoso e atencioso (Denier, 2008).

O desenvolvimento tecnológico na área da saúde caminha no sentido oposto ao padrão característico de outras áreas que consomem e desenvolvem tecnologias. Em geral, se há o aumento da demanda e consequentemente da produção de um determinado produto, o seu custo tende a cair. No caso da 
indústria farmacêutica e dos equipamentos utilizados para a atenção à saúde, independentemente do crescimento da sua utilização, o seu custo permanece alto, de forma que o interesse mercadológico torna consumidores os usuários dos serviços de saúde (Zucchi, Del Nero e Malik, 2000; Luz, 2005) - o que se relaciona com os achados do item 'uso de tecnologias voltadas para o aperfeiçoamento da atenção à saúde', classificação realizada no processo global dessa revisão sistemática, na medida em que alguns artigos foram classificados em mais de uma área. Sublinha-se aqui a correlação que os estudos sociológicos realizam entre cuidado e tecnologia.

Outra questão apresentada nos textos foi o crescimento do setor privado de saúde e as implicações das relações financeiras para a obtenção do cuidado. Dois textos abordaram de maneira oposta esse assunto. O primeiro postulou que nos ambientes privados de atenção à saúde as relações monetárias para o acesso ao cuidado são geradoras de descontentamento e conflito na relação entre médico e paciente. Como os domínios econômico e social são distintos, as relações financeiras no âmbito da saúde diferem de outros tipos de relação fornecedor/consumidor, e o dinheiro não é um meio neutro para estabelecer relações de interesse mútuo (Oh, 2013).

O segundo texto, de Samitt (2004), como contraponto descreve uma clínica particular que visa oferecer uma atenção ao paciente na mesma qualidade da Disney e do Hotel Ritz. O autor concebeu a qualidade como a primeira estratégia do mercado, ao considerar que a sociedade está cada vez mais voltada para um consumo aprimorado. A prestação de serviços na saúde, salienta, é uma indústria e um produto para consumo como outro qualquer, e é preciso investir não só na qualidade clínica como também na organização do atendimento, na estrutura do espaço e em tudo que dê a sensação de o cliente estar em um hotel de luxo. Esse texto tem como característica a propaganda de uma clínica particular, sendo apresentado aqui como referência, mesmo que caricatural, para demonstrar a existência da ideia da oferta de um cuidado em que a qualidade está na dimensão da materialidade da relação entre fornecedor e consumidor na aquisição de um produto, pautada nos valores da sociedade de consumo.

Ambos os textos demonstram a discussão classificada no todo da revisão no item 'fatores econômicos influenciando a atenção à saúde', ratificando a presença de fatores econômicos na conceituação e aplicação do cuidado, do ponto de vista sociológico. Ao aprofundar essa dimensão, Tronto (2007) relaciona cuidado, políticas públicas e democracia, ao assinalar a profundidade do debate entre elementos macro e microssociais.

Outra discussão em torno das disparidades sociais coaduna-se com a referência já estabelecida sobre os determinantes sociais de saúde (Buss, 2007) para a compreensão, em nível macro, do que efetivamente faz parte do cuidado 
em saúde. Todavia, esses apontamentos precisam ser contextualizados, pois podem induzir à conclusão de que a saúde deve 'resolver' as disparidades sociais. É preciso ressalvar que, da mesma forma que cuidado não é sinônimo de cuidado em saúde, as questões sociais não podem ser interpretadas como demandas de saúde, na medida em que implicam uma macroestrutura social mais ampla e atingem toda a configuração da vida dos sujeitos. O risco se dá em camuflar em problemas de saúde questões que são eminentemente sociais, o que pode vir a resultar na medicalização social, tornando o cuidado em tratamento de um adoecimento que não existe. Se a desigualdade social e, consequentemente, o sofrimento social forem compreendidos como sofrimento psíquico, por exemplo, podem-se desdobrar na produção social da loucura (Malfitano, Adorno e Lopes, 2011).

Uma abordagem que por vezes circunda as esferas sociais e de saúde, com possibilidades de produção da medicalização social, se dá no ambivalente monitoramento do risco, individualizando as explicações e, consequentemente, as ações de cuidado, em detrimento de sua dimensão coletiva (Fine, 2005). As noções envolvidas no controle do risco (Caponi, 2004) pertencentes ao modelo biomédico de cuidado, além de normatizarem a doença (Canguilhem, 2002) para atingir a ideia de prevenção, empreendem formas de controle da população. Tesser (2007) entende que a missão ética da biomedicina é de curar, tratar dos doentes e promover a saúde; no entanto, o seu saber está voltado para a cura ou o controle de doenças específicas, o que leva à conversão do doente em doença e da cura em controle de doenças e de riscos. Dessa maneira, ao focar na doença e não no sujeito que adoece, a clínica médica (Campos e Bedrikow, 2014), majoritariamente, ao exercer a hegemonia das ações de cuidado, se distancia de compreendê-lo do ponto de vista da relação entre sujeitos envolvidos em interações sociais. Entretanto, é preciso assinalar movimentos que buscam outros princípios de cuidados, pautados pela humanização e horizontalidade da assistência, por meio de uma clínica ampliada e contextualizada na vida dos sujeitos. Em outras palavras, advoga-se por uma análise sociológica que contribua para uma forma menos tecnificada e mais socializada de cuidado em saúde.

\section{Conclusão}

O material colhido na pesquisa aqui apresentada, mesmo com um número reduzido de artigos, forneceu elementos úteis para maior visibilidade da abordagem sociológica sobre o cuidado, notadamente por entender as ações de cuidado envolvendo dinamicamente os atores sociais, para além da aplicação de um procedimento técnico-tecnológico nos ambientes de assistência à saúde. O cuidado, conceitualmente, está na dimensão da solidariedade, do respeito e do zelo na relação entre sujeitos no esforço de ajuda mútua para 
a conservação da vida. Nesse sentido, ele pode ser compreendido como a base necessária para um resultado satisfatório na aplicação das mais variadas ações sociais, inclusive as de assistência à saúde.

A naturalização do termo cuidado, ao ser referenciado majoritariamente pelas ações efetuadas no campo da saúde, mais especificamente por meio de suas técnicas, fez com que se enfraquecesse o seu sentido mais básico e imprescindível. Esta afirmação não desconsidera os movimentos que advogam pela institucionalidade do cuidado de forma solidária e humanizada; pelo contrário, a discussão em tela pretende fortalecer tais iniciativas. Contudo, é preciso ressaltar a predominância de ações tecnificadas e avançar para o cuidado em sua dimensão social, de elo para uma comunicação solidária entre as pessoas, em que o cuidado ganha potência e suas ações tornam-se sensíveis às necessidades individuais e coletivas.

A potencialidade do saber profissional especializado do cuidado tem sido questionada e leva à criação de múltiplos conceitos de cuidado, que buscam ir além de ser apenas novos modelos de aplicação técnica de atenção à saúde. No entanto, pode-se postular que o alcance desses novos modelos sempre estará limitado à perspectiva social de quem o aplica. Se a ideia de cuidado continuar focada em uma ação específica, e não na sua dimensão solidária, sempre haverá a frustrante troca de procedimentos na tentativa de contemplar melhor as necessidades sociais dos atores envolvidos, sem nunca realmente alcançar uma relação entre pessoas que seja essencialmente cuidadora. O fracasso ou o sucesso do cuidar não está baseado apenas na sua qualidade técnica, mas sim na horizontalidade da relação entre sujeitos que se reconhecem parte da comunidade humana.

Portanto, o cuidado não está restrito à dimensão individual dos sujeitos. Os textos aqui analisados apresentam subsídios que permitem também abordá-lo como elo fundamental para a comunicação entre a macro e a microestrutura social. Ao compreendê-lo de forma mais ampla no interior da estrutura social, o cuidado passa a atuar tanto no cenário político quanto no individual, de maneira que as políticas sociais passam a ter, igualmente, a conotação de ações de cuidado. Entretanto, não se pode esquecer que, como nas relações interpessoais, o que qualifica as ações de cuidado, empreendidas pelo Estado ou por qualquer instituição, está nas bases do seu fundamento e não só na sua execução. Será determinante para o seu resultado a intenção cuidadora implícita na sua elaboração.

Tais apontamentos devem ser considerados por todas as áreas que empregam ações de cuidado em suas práticas cotidianas, como o campo da saúde, alargando sua compreensão de cuidado e empreendendo oficialmente outras discussões para a efetivação do cuidado. Evidencia-se, portanto, que o cuidado não está restrito ao campo da saúde. Fica perceptível que uma leitura sociológica do cuidado abrange as microrrelações entre indivíduos, assim como 
as macrorrelações entre o Estado e a população, o que poderia ser chamado de 'construção social do cuidado'. Assim, torna-se possível a concepção de um conceito de cuidado que não se restrinja a técnicas, tecnologias ou relação entre profissionais e pacientes, de modo a ampliar o cuidado para uma composição de elementos que inclua questões econômicas, sociais e políticas na sua constituição.

Faz-se necessário um aprofundamento de uma sociologia do cuidado e, no caso da literatura brasileira, seu desenvolvimento com maior predominância no campo acadêmico, transcendendo o campo da saúde, mesmo quando referenciada nele. Ou seja, que o cuidado possa ganhar maior visibilidade nos estudos por meio de sua compreensão sociológica, mesmo quando delimitado ao setor saúde. Se a qualidade da interação social entre sujeitos, no âmbito das redes sociais comunitárias ou relações profissionais especializadas, interfere fortemente nos efeitos da atenção à saúde, não se pode falar de cuidado sem no mínimo falar de democracia, justiça social, solidariedade, política social, capacidade de acesso aos bens sociais e dos tensionamentos e restrições produzidas pelos fatores econômicos. Estes são pontos que teriam maior visibilidade com a ampliação de estudos na dimensão sociológica do cuidado, a qual potencialmente pode contribuir para a melhoria da estruturação das ações formais e informais voltadas à manutenção da vida. Advoga-se, também, que maior visibilidade da dimensão sociológica do cuidado nas discussões brasileiras poderá contribuir para suas ações em diferentes campos, incluindo o cuidado em saúde.

\section{Colaboradores}

Octávio Augusto Contatore contribuiu para o levantamento, a discussão e a análise dos dados e é autor da dissertação que gerou o presente artigo; Ana Paula Serrata Malfitano foi responsável pela discussão dos dados, redação e revisão do texto; Nelson Filice de Barros foi responsável pela orientação da pesquisa, redação e revisão do texto. Todos os autores aprovaram a versão final do artigo. 


\section{POR UNA SOCIOLOGIA DEL CUIDADO: REFLEXIONES PARA MÁS ALLÁ DEL CAMPO DE LA SALUD}

Resumen Mientras la encuesta sobre el cuidado esté predominantemente asociada al campo de la salud, los estudios sociológicos resaltan que los elementos que lo influencian no se restringen a él, en la medida en que es la expresión de apoyo social intenso y atributo fundamental para el mantenimiento de la vida colectiva. El objetivo es hipervisibilizar la dimensión sociológica del cuidado para problematizar su transposición como factor necesario para la vida social para una acción técnica de salud. Se realizó una revisión sistemática de literatura, entre 2003 y 2013, en la Biblioteca Virtual en Salud, PubMed, Scopus, Embase, Web of Science, Francis (Ovid), ProQuest Central, AcademicSearch Premier (EBSCOhost), Jstor y Sage, con el unitérmino cuidado y variantes. De 262 artículos identificados, fueron seleccionados 15 sobre las dimensiones sociales del cuidado. Se observó que el cuidado aludió la solidaridad y calidad de la interacción entre sujetos en las microrrelaciones y en las macroestructuras, diferenciándose de su aplicación técnica/tecnológica en salud; y también que es más abarcativo definirlo sociológicamente que por la especificidad de un acto, pues incluye dimensiones de solidaridad, respecto, entusiasmo y ayuda mutua para la conservación de la vida. Se concluyó que un abordaje sociológico del cuidado es necesario, contribuyendo inclusive al campo de la salud.

Palavras clave sociología; cuidados; cuidados de salud; apoyo social.

\section{Nota}

${ }^{1} \mathrm{~A}$ Embora seja um número reduzido de textos, optou-se pela sua análise integral na busca de explicitar o que a produção sociológica tem abordado sobre o cuidado. Destaca-se que este artigo integra pesquisa maior, voltada para a discussão do cuidado, sendo que outras produções se voltaram para a especificidade da análise dos artigos sobre o cuidado em saúde. 


\section{Referências}

ALMEIDA, Patty F.; MARIN, Juliana; CASOTTI, Elisete. Estratégias para consolidação da coordenação do cuidado pela atenção básica. Trabalho, Educação e Saúde, Rio de Janeiro, v. 15, n. 2, p. 373-398, ago. 2017.

AYRES, José R. C. M. Cuidado e reconstrução das práticas de saúde. Interface: Comunicação, Saúde e Educação, Botucatu, v. 8, n. 14, p. 73-92, 2004.

BARROS, Nelson F. As práticas integrativas e complementares como cuidado emancipador: gestão de práticas integrativas e complementares em saúde. Disponível em: <https://avasus.ufrn.br/ local/avasplugin/cursos/curso.php?id=151>. Acesso em: 12 set 2018.

BEAUVOIR, Simone. O segundo sexo. v. I e II. Rio de Janeiro: Nova Fronteira, 1980.

BIASOLI-ALVES, Zélia M. M. Continuidades e rupturas no papel da mulher brasileira no século XX. Psicologia: Teoria e Pesquisa, Brasília, v. 16, n. 3, p. 233-239, 2000.

BRASIL. Ministério da Saúde. Diretrizes para a organização das redes de atenção à saúde do SUS. Brasília: Ministério da Saúde, 2010.

BRASIL. Ministério da Saúde. Portaria n. 3.088/GM/MS, de 23 de dezembro de 2011. Institui a Rede de Atenção Psicossocial para pessoas com sofrimento ou transtorno mental, incluindo aquelas com necessidades decorrentes do uso de álcool, crack e outras drogas no âmbito do SUS. Diário Oficial da União, Brasília, DF, 30 dez. 2011, Seção 1, p. 59-61.

BUSS, Paulo M. Globalização, pobreza e saúde. Ciência \& Saúde Coletiva, Rio de Janeiro, v. 12, n. 6, p. 1.575-1.589, 2007.

CAILlÉ, Alain. Dádiva, care e saúde. Sociologias, Porto Alegre, v. 16, n. 36, p. 42-59, 2014.

CAMARGO JR., Kennedy R. A biomedicina. Physis: Revista de Saúde Coletiva, Rio de Janeiro, v. 15, p. 177-201, 2005.
CAMPOS, Gastão W. S.; BEDRIKOW, Rubens. História da clínica e atenção básica: o desafio da ampliação. São Paulo: Hucitec, 2014.

CANESQUI, Ana M.; BARSAGLINI, Reni A. Apoio social e saúde: pontos de vista das ciências sociais e humanas. Ciência \& Saúde Coletiva, Rio de Janeiro, v. 17, n. 5, p. 1.103$1.114,2012$.

CANGUILHEM, Georges. O normal e o patológico. 5. ed. Rio de Janeiro: Forense Universitária, 2002.

CAPONI, Sandra. A saúde como abertura ao risco. In: CZERESNIA, Dina; MACHADO, Carlos (orgs.). Promoção da saúde. 3. ed. Rio de Janeiro: Editora Fiocruz, 2004. p. 55-77.

CARPENTIER, Normand. Entry into a care trajectory: individualization process, networks, and the emerging project. SAGE Open, v. 3, n. 2, p. 1-9, 2013.

CARPENTIER, Normand; GRENIER, Amanda. Successful linkage between formal and informal care systems: the mobilization of outside help by caregivers of persons with Alzheimer's disease. Qualitative Health Research, v. 22, n. 10, p. 1.330-1.344, 2012.

CASTEL, Robert. Da indigência à exclusão, a desfiliação: precariedade do trabalho e vulnerabilidade relacional. In: LANCETTI, Antônio (org.). Saúde e loucura 4. São Paulo: Hucitec, 1994. p. 21-48.

CECILIO, Luiz C. O. Apontamentos teóricoconceituais sobre processos avaliativos considerando as múltiplas dimensões da gestão do cuidado em saúde. Interface: Comunicação, Saúde e Educação, Botucatu, v. 15, n. 37, p. 589-599, 2011.

CLEMENTS, Archie et al. Overcrowding and understaffing in modern health-care systems: key determinants in meticillin-resistant Staphylococcus aureus transmission. The Lancet Infectious Diseases, v. 8, n. 7, p. 427-434, 2008. 
CONTATORE, Octávio A.; MALFITANO, Ana P. S.; BARROS, Nelson F. Os cuidados em saúde: ontologia, hermenêutica e teleologia. Interface: Comunicação, Saúde e Educação, Botucatu, v. 21, n. 62, p. 553-563, 2017.

CONTATORE, Octávio A. Os sentidos dos cuidados em saúde. 2016. 122f. Dissertação (Mestrado em Saúde Coletiva) - Universidade Estadual de Campinas, Faculdade de Ciências Médicas, Campinas, 2016.

COSTA, Ana A. A.; SARDENBERG, Cecília M. B. (orgs.). Feminismo, ciência e tecnologia. Salvador: Redor/Núcleo de Estudos Interdisciplinares sobre a Mulher/Faculdade de Filosofia e Ciências Humanas da Universidade Federal da Bahia, 2002. Disponível em: < http://www. neim.ufba.br/site/arquivos/file/feminismocienciencia.pdf $>$. Acesso em: 9 mar. 2018.

DEBERT, Guita G. Arenas de conflito em torno do cuidado. Tempo Social, São Paulo, v. 26, n. 1, p. 35-45, 2014.

DENIER, Yvonne. Mind the gap! Three approaches to scarcity in health care. Medicine, Health Care and Philosophy, v. 11, n. 1, p. 73-87, 2008.

DOVIDIO, John F. et al. Disparities and distrust: the implications of psychological processes for understanding racial disparities in health and health care. Social Science and Medicine, v. 67, n. 3, p. 478-486, 2008.

EPELE, María. Sobre o cuidado de outros em contextos de pobreza, uso de drogas e marginalização. Mana, Rio de Janeiro, v. 18, n. 2, p. 247-268, 2012.

ERDMANN, Alacoque L. et al. As organizações de saúde na perspectiva da complexidade dos sistemas de cuidado. Revista Brasileira de Enfermagem, Brasília, v. 57, n. 4, p. 467471, 2004.

FERLANDER, Sara. The importance of different forms of social capital for health. Acta Sociologica,, v. 50, n. 2, p. 15-128, 2007.
FINE, Michael. Individualization, risk and the body: Sociology and care. Journal of Sociology, v. 41, n. 3, p. 247-266, 2005.

FOUCAULT, Michel. O nascimento da clínica. Rio de Janeiro: Forense Universitária, 1977.

GEORGES, Isabel P. H.; SANTOS, Yumi Garcia. Olhares cruzados: relações de cuidado, classe e gênero. Tempo Social, São Paulo, v. 26, n. 1, p. 47-60, 2014.

GOMIDE, Márcia; GROSSETTI, Michel. Rede social e desempenho de programas de saúde: uma proposta investigativa. Physis: Revista de Saúde Coletiva, Rio de Janeiro, v. 20, n. 3, p. 873-893, 2010.

GONÇALO, Camila da Silva et al. Planejamento e execução de revisões sistemáticas da literatura. Brasília Médica, Brasília, v. 49, p. 104-110, 2012.

GUIMARÃES, Nadya A.; HIRATA, Helena S.; SUGITA, Kurumi. Cuidado e cuidadoras: o trabalho de care no Brasil, França e Japão. Sociologia \& Antropologia, Rio de Janeiro, v. 1, n. 1, p. 151-180, 2011.

HANLON, Neil; HALSETH, Greg. The greying of resource communities in northern British Columbia: implications for health care delivery in already-underserviced communities. Canadian Geographer, v. 49, n. 1, p. 1-24, 2005.

HOLLAN, Douglas. Being there: on the imaginative aspects of understanding others and being understood. Ethos, v. 36, n. 4, p. 475489, 2008.

ILLICH, Ivan. A expropriação da saúde: nêmesis da medicina. 3. ed. Rio de Janeiro: Nova Fronteira, 1975.

KIM, Jim Y.; FARMER, Paul; PORTER, Michael E. Redefining global health-care delivery. The Lancet, v. 382, n. 9.897, p. 1.060-1.069, 2013.

LALLEMENT, Michel. Comparações internacionais: desafios para os estudos sobre trabalho 
e gênero. Tempo Social, São Paulo, v. 26, n. 1, p. 147-161, 2014.

LOPES, Andréia A. F. Cuidado e empoderamento: a construção do sujeito responsável por sua saúde na experiência do diabetes. Saúde e Sociedade, São Paulo, v. 24, n. 2, p. 486-500, 2015.

LUZ, Madel T. Medicina e racionalidades médicas: estudo comparativo da medicina ocidental contemporânea, homeopática, chinesa e ayurvédica. In: CANESQUI, Ana M. (org.). Ciências sociais e saúde para o ensino médico. São Paulo: Hucitec, 2000. p. 181-200.

LUZ, Madel T. Cultura contemporânea e medicinas alternativas: novos paradigmas em saúde no fim do século XX. Physis: Revista de Saúde Coletiva, Rio de Janeiro, v. 15, supl., p. 145-176, 2005.

MALFITANO, Ana P. S.; ADORNO, Rubens C. F.; LOPES, Roseli E. Um relato de vida, um caminho institucional: juventude, medicalização e sofrimentos sociais. Interface: Comunicação, Saúde e Educação, Botucatu, v. 15, n. 38, p. 701-714, 2011.

MINAYO, Maria C. S. O desafio do conhecimento: pesquisa qualitativa em saúde. 10. ed. São Paulo: Hucitec, 2007.

MOLINIER, Pascale. Cuidado, interseccionalidade e feminismo. Tempo Social, São Paulo, v. 26, n. 1, p. 17-33, 2014.

MURRAY, Marjorie et al. Care and relatedness among rural Mapuche women: issues of carino and empathy. Ethos, v. 45, n. 3, p. $367-385,2017$.

NOONE, Jack H.; STEPHENS, Christine. Men, masculine identities, and health care utilisation. Sociology of Health and Illness, v. 30, n. 5, p. 711-725, 2008.
$\mathrm{OH}$, Hyeyoung. Consumerism in action: how patients and physicians negotiate payment in health care. Qualitative Health Research, v. 23, n. 3, p. 385-395, 2013.

PAVLOKOVÁ, Katerine; PRUSVIC, David. Generational accounting in European health care systems. Czech Journal of Economics and Finance, v. 60, n. 5, p. 378-399, 2010.

PESCOSOLIDO, Bernice A. Of pride and prejudice: the role of Sociology and social networks in integrating the health sciences. Journal of Health and Social Behavior, v. 47, n. 3, p. 189-208, 2006.

PIRES, Maria R. G. M.; DEMO, Pedro. Políticas de saúde e crise do estado de bem-estar: repercussões e possibilidades para o Sistema Único de Saúde. Saúde e Sociedade, São Paulo, v. 15, n. 2, p. 56-71, 2006.

SAMITT, Craig E. At your service: transforming health care delivery. Physician Executive, v. 30, n. 6, p. 36-39, 2004.

SANTOS, Boaventura S. A crítica da razão indolente: contra o desperdício da experiência. 4. ed. São Paulo: Cortez, 2002.

SILVA, Maria N. R. M. O. et al. Desenvolvendo e articulando a rede intersetorial para cuidado integral de usuários de drogas em contextos de vulnerabilidade. Cadernos Brasileiros de Terapia Ocupacional, São Carlos, v. 22, suplemento especial, p. 145-152, 2014.

SORJ, Bila. Socialização do cuidado e desigualdades sociais. Tempo Social, São Paulo, v. 26, n. 1, p. 123-128, 2014.

SPATES, Kamesha. "The missing link": the exclusion of black women in psychological research and the implications for black women's mental health. SAGE Open, v. 2, n. 3, p. 1-8, 2012. 
TESSER, Charles D. A verdade na biomedicina, reações adversas e efeitos colaterais: uma reflexão introdutória. Physis: Revista de Saúde Coletiva, Rio de Janeiro, v. 17, n. 3, p. 465-484, 2007.

TRONTO, Joan. Assistência democrática e democracias assistenciais. Sociedade e Estado, Brasília, v. 22, n. 2, p. 285-308, 2007.
ZUCCHI, Paola; DEL NERO, Carlos; MALIK, Ana M. Gastos em saúde: os fatores que agem na demanda e na oferta dos serviços de saúde. Saúde e Sociedade, São Paulo, v. 9, n. 1-2, p. 127-150, 2000. 
DOI: http://dx.doi.org/10.1590/1981-7746-sol00200

\section{Errata}

No artigo POR UMA SOCIOLOGIA DO CUIDADO: REFLEXÕES PARA ALÉM DO CAMPO DA SAÚDE, autoria de Octávio Augusto Contatore, Ana Paula Serrata Malfitano e Nelson Filice de Barros, com número de DOI: 10.1590/1981-7746-sol00175, publicado no periódico Trabalho, Educação e Saúde, v. 17, n. 1, identificador eletrônico 17(1):e0017507, página 1, onde se lia:

“POR UMA SOCIOLOGIA DO CUIDADO: REFLEXÕES PARA ALÉM DO CAMPO"

LEIA-SE:

“POR UMA SOCIOLOGIA DO CUIDADO: REFLEXÕES PARA ALÉM DO CAMPO DA SAÚDE”

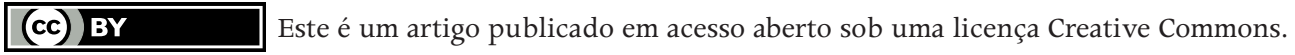

